\title{
Temporal isolation effects in recognition and serial recall
}

\author{
Caroline Morin \\ Cranfield University, Swindon, England \\ Gordon D. A. Brown \\ University of Warwick, Coventry, England \\ and University of Western Australia, Nedlands, Western Australia, Australia \\ AND \\ STEPHAN LEWANDOWSKY \\ University of Western Australia, Nedlands, Western Australia, Australia
}

\begin{abstract}
Recent temporal distinctiveness models of memory predict that temporally isolated items will be recalled better than temporally crowded items. The effect has been found in some tasks (free recall, memory for serial order when report order is unconstrained, running memory span) but not in others (forward serial recall). Such results suggest that the attentional weighting given to a temporal dimension in memory may vary with task demands. Here, we find robust temporal isolation effects in recognition memory (Experiment 1) and a smaller isolation effect in forward serial recall when an open pool of items is used (Experiment 2). Analysis of 26 temporal isolation effects suggests that the phenomenon occurs in a range of tasks but is larger when it is useful to attend to a temporal dimension in memory. The overall pattern of results is taken to favor memory models that rely on multiple weighted dimensions in memory, one of which is temporal.
\end{abstract}

According to temporal distinctiveness models of memory, items are represented in memory partly in terms of their locations along a temporal dimension. Such models predict that memory traces of items that were temporally separated from their neighbors during presentation will occupy relatively isolated positions along the temporal dimension in memory. These memory traces will be more discriminable from each other than will traces for temporally crowded items and, hence, will be easier to retrieve (e.g., Brown, Neath, \& Chater, 2007; Brown, Preece, \& Hulme, 2000). In contrast, a number of other models allocate no role (or a more limited role) to time during the encoding of list items (e.g., Farrell \& Lewandowsky, 2002; Lewandowsky \& Murdock, 1989; Oberauer \& Lewandowsky, 2008; Page \& Norris, 1998), and such models typically predict that temporal isolation effects should not occur. The present study tested the predictions of temporal distinctiveness models in a recognition memory task and in a forward serial recall task that used an open pool of items. Both experiments examined memory for verbal materials and used a continual distractor task to prevent rehearsal.

Crowder's (1976) telephone pole analogy illustrates the key idea of temporal distinctiveness models (Bjork $\&$ Whitten, 1974). At the time of recall, items that were evenly spaced during presentation can be seen as analogous to telephone poles receding into the distance from the viewpoint of a stationary observer. Early-list items are analogous to distant telephone poles and are less discriminable, whereas recently presented items are more easily discriminable (analogously to the closer telephone poles). Thus, a key idea is that crowdedness along a temporal dimension in memory is closely related to discriminability and, hence, to recall probability.

In the telephone pole example, recent items are assumed to have less crowded and, hence, more discriminable memory representations because they are recent; the crowdedness arises from the relationship between the interitem interval and the retention interval. Thus, the relative advantage of recent items will be reduced as time passes (e.g., when there is a filled retention interval), and their temporal discriminability will be reduced. One recent model (SIMPLE; Brown et al., 2007) captures this effect of time on memory by postulating that the confusability of any two items in memory is a function of the ratio of their temporal distances at the time of recall (the smaller divided by the larger). For example, consider two items, one of which occurred $2 \mathrm{sec}$ in the past and the other of which occurred $4 \mathrm{sec}$ in the past. The temporal distance ratio of these two items will be $2 / 4=.5$. Now consider in contrast the same two items after a further $10 \mathrm{sec}$ has passed. The items' temporal distances will now be 12 and $14 \mathrm{sec}$, and their confusability will be $12 / 14=.86$. Thus, the confus-

C. Morin, c.morin@cranfield.ac.uk 
ability between the item memories has increased from .5 to .86 as a result of the increased retention interval.

Temporal distinctiveness models such as SIMPLE can therefore capture the fact that memory performance depends in predictable ways on the relation between interitem intervals and retention intervals and have been applied, in particular, to the interaction of serial position curves with retention interval (see, e.g., Brown \& Lewandowsky, 2010; Knoedler, Hellwig, \& Neath, 1999; Nairne, Neath, Serra, \& Byun, 1997; Neath, 1993a, 1993b; Neath $\&$ Knoedler, 1994). Further evidence that temporal discriminability affects memorability is the fact that serial position curves (for memory for verbal items) can be predicted from the discriminability (in an absolute identification task) of temporal durations corresponding to the retention intervals for the individual items (Brown, Vousden, \& McCormack, 2009).

Most experiments on temporal distinctiveness have focused on how the discriminability of items in memory changes as a function of retention interval and interitem gap. Experimental manipulations of temporal distinctiveness in such experiments have typically involved presentation rate (all interitem gaps are changed) and/or retention interval. However, according to temporal distinctiveness models of memory, it should be possible to change the discriminability of an item in memory in a different waynamely, by increasing the temporal gaps surrounding that individual item. Specifically, the introduction of longer temporal gaps before and after an item during its presentation should (even if rehearsal is prevented) make that item easier to discriminate from its neighbors in psychological space and, hence, make it easier to recall. We refer to such effects as temporal isolation effects (TIEs). For example, in the sequence A.B.C...D...E., where dots represent the passage of time, $\mathrm{D}$ is temporally isolated. Note that we reserve the term TIE for cases in which the gaps surrounding individual items are manipulated experimentally; the memory advantage that an item may have through having been presented recently is assumed to arise from the same mechanism (temporal distinctiveness) but does not fall within our definition of a TIE.

Empirical studies do not always show TIEs, and their presence seems to depend in predictable ways on task demands. In particular, studies of immediate forward serial recall have typically failed to show unambiguous TIEs, whereas studies involving other modes of recall often have shown clear TIEs. To foreshadow our conclusions, the methodological details appear to matter, and they matter in ways that can be understood from an adaptive perspective. Here and throughout, we focus only on studies involving memory for verbal material, while noting the existence of studies that have also shown TIEs in memory for nonverbal material (e.g., Guérard, Neath, Surprenant, \& Tremblay, 2010).

First we consider immediate forward serial recall. In this paradigm, participants not only must recall information about the serial order of presented items, but also must recall that information in the order of its presentation (i.e., the first-presented item must be the first recalled or placed in its correct serial position). Initial studies showed that TIEs emerge when the schedule of presentation is predictable (increasing vs. decreasing schedules of presentation; Crowder \& Neath, 1991; Lewandowsky, Wright, \& Brown, 2007; Neath \& Crowder, 1990, 1996; see also Corballis, 1966; Schonfield \& Donaldson, 1966; Welte \& Laughery, 1971). For example, performance is relatively better at the start of a list when rate of presentation increases throughout a list (because the temporal gaps surrounding the early-list items are larger than the temporal gaps surrounding late-list items). However, Lewandowsky et al. (2007) argued that the effect observed when presentation schedule is predictable does not provide unequivocal evidence for a causal effect of temporal isolation, because (1) subvocal rehearsal was not prevented in several of the early studies (although Neath \& Crowder, 1996, used a sufficiently fast rate of presentation to render rehearsal unlikely) and (2) strategic effects might have occurred such that participants selectively encoded the items that were presented relatively slowly within a list. The latter possibility was confirmed by Lewandowsky et al. (2007) in a simulation that showed that the effects of a predictable presentation schedule could be produced by a model in which only encoding varied (such that the more widely spaced items were assumed to have a higher probability of being encoded).

Consistent with such an interpretation, when the interitem temporal intervals have been randomly presented within each list, most studies have not shown any effect of temporal isolation in forward serial recall (see Geiger \& Lewandowsky, 2008, for a summary table). This failure to find a TIE in forward serial recall was reported by Lewandowsky and Brown (2005), and no TIE has been found for either heard or seen events (Nimmo \& Lewandowsky, 2005), with brief gaps or very long pauses between items (Nimmo \& Lewandowsky, 2006), with auditory verbal and spatial materials (Parmentier, King, \& Dennis, 2006), with probed recall (Lewandowsky, Brown, Wright, \& Nimmo, 2006), and with a serial and temporal recognition task (Farrell \& McLaughlin, 2007; although see Farrell, 2008). Such results were taken as support for the suggestion that forward serial recall fosters reliance on a positional, rather than a temporal, dimension to encode the order of items (e.g., Lewandowsky, Duncan, \& Brown, 2004). The results might, nonetheless, be compatible with distinctiveness models if it is assumed that people have access to both a positional and a temporal dimension in memory space (Brown et al., 2007) and that they may shift their attention between dimensions in response to task demands.

Support for the idea that temporal information is encoded in memory and utilized at retrieval comes from the fact that TIEs do emerge in a range of memory tasks other than forward serial recall. Brown, Morin, and Lewandowsky (2006) found a significant effect of temporal isolation with random presentation schedules in free recall. Geiger and Lewandowsky (2008) found TIEs in a running memory span task - that is, a task in which list length was unpredictable and people had to recall the last four items of the list in forward order. Lewandowsky, Nimmo, and Brown (2008) found that when report order in a serial order reconstruction task was unconstrained (i.e., participants were allowed 
to reconstruct the list items in an order of their choosing), a clear TIE emerged. In contrast, when report order was constrained (i.e., participants were required to reconstruct the order of the list in presentation order), no TIE was found (consistent with the serial recall studies reviewed above). In summary, TIEs are observed in a range of memory tasks other than immediate forward serial memory.

At first glance, the fact that TIEs do not emerge with serial recall might suggest that no temporal information is encoded when items are presented for forward serial recall. However, such a conclusion would be premature. Lewandowsky, Nimmo, and Brown (2008) found that the differences between forward and unconstrained report remained even when participants were unable (due to postcuing of report order) to anticipate the type of test at encoding. Thus, temporal information was demonstrably available but not used when reconstruction was in forward order. Lewandowsky, Nimmo, and Brown concluded that (1) both positional and temporal information are inevitably encoded but (2) the relative weighting between the two dimensions can be altered at recall.

Here, we additionally hypothesize that the greater reliance on a temporal dimension when recall order is unconstrained is adaptive (see also Lewandowsky, Brown, \& Thomas, 2009), because, if recent items are recalled first, they are advantaged by their recency along the temporal dimension (cf. the telephone pole analogy).

We now turn to the case of recognition memory, in which TIEs have not yet been studied. What predictions can be made for TIEs in this task? In a recognition task in which memory is probed immediately after list presentation, recent items will be advantaged if attention is allocated to a temporal dimension at retrieval. According to the adaptive approach, therefore, recency effects and TIEs should also be found in a recognition memory task. Given that recency effects are already known to occur in recognition when testing conditions are appropriate (Talmi \& Goshen-Gottstein, 2006), Experiment 1 examined the effects of temporal isolation in a recognition memory task.

A second goal of the present study was to reexamine the failure to find an isolation effect in forward serial recall under conditions that differed in theoretically important ways from those in previous studies. First, it is possible that TIEs in the forward serial recall studies reviewed earlier were masked by rehearsal processes occurring during the temporal gaps between items. Specifically, longer temporal intervals surrounding a given item might be used to rehearse previously presented list items and, hence, reduce the effective isolation of the given item, as well as allowing compensatory rehearsal of earlier, perhaps less isolated, items. Although the earlier studies used articulatory suppression to prevent subvocal rehearsal, Lewandowsky, Geiger, and Oberauer (2008) found that the articulation of changing, rather than repeated, distractors during recall had a substantially greater detrimental effect on performance (see also Jones \& Macken, 1993; Macken \& Jones, 1995). It follows that the repetitive articulation of a single distractor in the earlier studies may not have precluded at least some rehearsal. We therefore examined whether a more stringent rehearsal prevention manipulation, involving articulation of changing distractors, might allow TIEs to be observed even in forward serial recall.

Finally, with the exception of experiments using predictable presentation schedules, the studies of forward serial recall above, which did not show an effect of temporal isolation, used a closed pool of items (the same limited set of letters was used throughout the experiment). It is possible that this methodological choice may have highlighted the importance of order information at the expense of item information, thus fostering greater reliance on a positional, rather than a temporal, dimension. We therefore examined whether the use of a large open pool (common English words) might decrease reliance on a positional dimension even in forward serial recall, which, in turn, might lead to the emergence of a TIE.

A third goal was to attempt something of an overview of TIEs obtained in published studies to date, with a view to identifying overall patterns and achieving some closure on TIEs in memory.

\section{EXPERIMENT 1}

The aim of the first experiment was to explore the hypothesis that TIEs emerge in memory tasks in which temporal recency may be used advantageously. Specifically, Experiment 1 used a recognition memory task with a continual distractor procedure during encoding to prevent rehearsal in the temporal gaps between items. Note that strong recency effects are seen in recognition memory tasks when memory is tested immediately after list presentation and only one item is tested on each list (Talmi \& Goshen-Gottstein, 2006). A known strong feature of temporal distinctiveness models of memory is their ability to account for recency effects under a wide variety of circumstances, so any finding of strong recency combined with an absence of TIEs would be problematic for such models.

In addition, Experiment 1 used a remember/know procedure in an attempt to separate recollection-based memory processes from familiarity-based processes (e.g., Yonelinas, 2002). Although the idea that remember and know responses reflect qualitatively different memory retrieval processes is by no means universally accepted (see, e.g., Dunn, 2004; Wixted, 2007), remember responses are sometimes thought to reflect the same recollection processes as those involved in free recall tasks, in which a TIE has already been found. It was therefore thought possible that TIEs would be more likely to be found for remember than for know responses.

\section{Method}

Participants. Twenty-four students from the University of Warwick participated voluntarily and received either course credit or a small payment (£5). The experiment lasted approximately $50 \mathrm{~min}$.

Materials. The experiment was controlled by E-Prime. One thousand sixty-four one- and two-syllable words with an average written frequency of $61(S D=109)$ and an average concreteness rating of $484(S D=110)$ were taken from the MRC Psycholinguistic Database (Wilson, 1988). From these 1,064 words, 112 lists of 9 words were constructed (1,008 words for the lists and 56 words as lures). A single probe item was presented at the end of each list. There were 
4 practice trials ( 2 positive and 2 negative) and 108 experimental trials. Fifty-four of those were positive trials on which the probe word had been presented in the list. Across trials, each serial position was tested six times. The other 54 trials were negative trials on which the probe was absent from the list. Positive and negative trials were randomly interleaved. During list presentation, each word was chosen randomly, without replacement, from the pool of stimulus words, and every word in the list (except for the last one) was followed by an interval (chosen randomly without replacement) that included one, two, three, four, five, six, or seven randomly chosen nonrepeating digits. Thus, all of the eight possible gaps appeared once and only once in each list.

Each trial began with the presentation of a cross in the middle of the screen for 2,000 msec, after which the first word was displayed. The words and digits were presented at a uniform rate of $550 \mathrm{msec} /$ item and had to be read aloud (i.e., digit shadowing served as a distractor activity). After the last list item, a blank screen was presented for $400 \mathrm{msec}$, and then the probe word was displayed between two rows of question marks. The participants' task was to indicate by a single keypress response whether (1) they knew that the item had not been presented on the list (no), (2) they remembered that the item had been presented (remember), or (3) they knew that the item had been presented but did not have a specific recollection of its occurrence (know). Presentation of each list lasted $22.75 \mathrm{sec}$ in total.

Procedure. The participants were tested individually in a soundattenuating booth. The experimenter was present throughout the experiment to ensure that the participants read the words and the digits aloud during presentation of the list.

\section{Results}

Figure 1 shows the observed proportions of remember, know, and no responses to old items as a function of serial position, as well as performance on the lures. As can be seen in the figure, remember responses increased for the last few serial positions, whereas know and no responses to old items decreased. For each participant, the slope of the function relating serial position (from Positions 6-9) and the three different types of responses (remember, know, and no) was calculated. The individual slope estimates were then subjected to an aggregate analysis. The recency slope for the remember responses was positive and significantly different from zero $[M=.11, S D=.08 ; t(23)=$ $6.89, p<.001, d=1.41]$, whereas the slopes for know responses $[M=-.08, S D=.08 ; t(23)=-4.79, p<.001$, $d=0.98]$ and $n o$ responses $[M=-.03, S D=.04 ; t(23)=$ $-3.66, p=.001, d=0.75]$ were also significantly different from zero but negative: As serial position increased, performance on these indicators decreased. The same analyses were also performed on the entire serial position curve (Positions 1-9) and showed the same pattern, with a positive slope for the remember responses $[M=.07, S D=$ $.03 ; t(23)=12.45, p<.001, d=2.54]$ and negative slopes for the know responses $[M=-.04, S D=.03 ; t(23)=$ $-7.40, p<.001, d=1.51]$ and $n o$ responses $[M=-.03$, $S D=.02 ; t(23)=-7.41, p<.001, d=1.51]$.

We considered remember and know responses separately for the next series of analyses, because the remember responses might be more likely to reflect the operation of a recall-based (rather than a familiarity-based) process of the type expected to be influenced by temporal isolation. Serial Positions 2-7 were used for the analysis, since Positions 1 and 9 were not preceded or followed by a gap. Position 8 was not included in the analysis, since performance was at ceiling in the overall accuracy and the remember responses. The high accuracy at this position might have masked any effect of temporal isolation.

As in the previous literature (e.g., Lewandowsky et al., 2006; Lewandowsky, Nimmo, \& Brown, 2008), we added

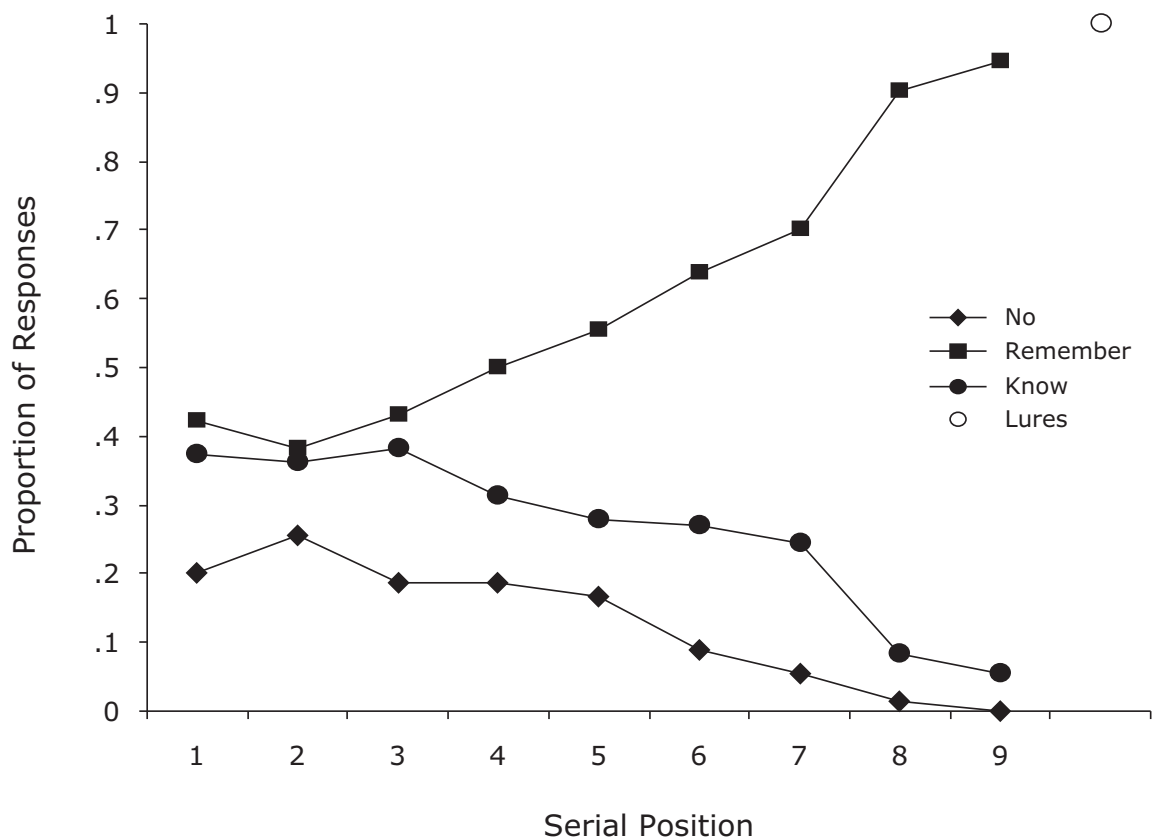

Figure 1. Proportions of remember, know, and no responses as a function of serial position in Experiment 1, and performance on the lures. 
preitem and postitem gaps to obtain a combined temporal isolation for each item. In order to ensure a sufficient number of data points, the 13 possible values of combined temporal isolation were collapsed into five groups containing a similar number of observations (specifically, temporal isolations of 1-3, 4 and 5, 6 and 7, 8 and 9, and finally $10-13$ were grouped together, where the figures refer to the number of digits, each lasting $550 \mathrm{msec}$ ).

As can be seen in Figure 2 (top line), remember responses increased with temporal isolation, rising from around $48 \%$ to around $58 \%$ for the longest combined gap. For each participant, the slope of the function relating remember responses to temporal isolation was calculated. The individual slope estimates were then subjected to an aggregate analysis. The mean slope for the remember responses was significantly different from zero $[M=.02$, $S D=.04 ; t(23)=2.31, p=.03, d=0.49]$. That is, for each additional second of temporal isolation, performance as measured by remember responses increased by around $2 \%$. The same analysis was carried out for overall accuracy and for know responses alone. The mean slopes for overall accuracy $[M=-.005, S D=.02 ; t(23)=1.26$, $p=.22, d=0.257]$ and know responses $[M=-.006$, $S D=.024 ; t(23)=1.16, p=.26, d=0.24]$ were not significantly different from zero.

Logistic regressions were also performed on the ungrouped data for each participant, using the combined temporal isolations prior to grouping of similar isolations and the time from item offset (the interval in milliseconds between the offset of the word and the beginning of the recall period) as predictors. The score for each response ( 0 or 1 , with 1 referring to either remember or know responses in separate analyses) was used as the dependent variable. The individual estimates were then subjected to an aggregate analysis. This analysis showed that the effect of temporal isolation on remember responses was still significant when time from item offset was partialed out $[M=.06, S D=.12 ; t(23)=2.40, p=.03, d=0.49]$. Likewise, the analyses on the know responses [M=-.02, $S D=.10 ; t(23)=-0.75, p=.46, d=0.15]$ and overall accuracy $[M=.06, S D=.21 ; t(23)=1.5, p=.15, d=$ $0.31]$ showed the same pattern as the collapsed data, with no significant effect of total isolation when time since item offset was partialled out.

\section{Discussion}

Results showed that TIEs were present in a recognition task but were limited to remember responses: No isolation effect emerged for know responses. These results were obtained when the schedule of presentation was randomized and rehearsal was minimized using a continual distractor task. We note the difficulty of ensuring that all forms of rehearsal were completely prevented, but also note that the distracting activity filled up all the time between items, thus not allowing any time for refreshing of memory traces (see Barrouillet, Bernardin, Portrat, Vergauwe, \& Camos,

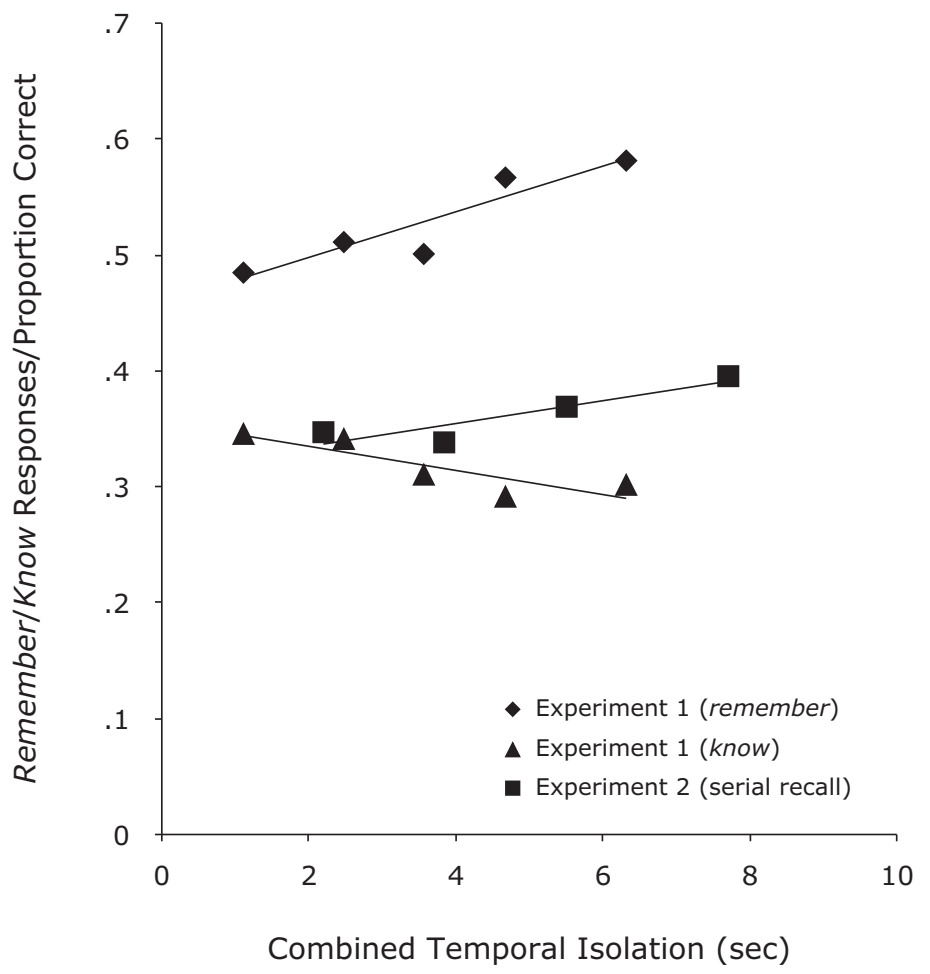

Figure 2. Proportions of remember and know responses as a function of binned total temporal isolation in Experiment 1 (recognition), and mean accuracy as a function of binned total temporal isolation in Experiment 2 (serial recall). 
2007). Moreover, the methodology used in Experiment 1 was slightly different from that used in other studies, in that the distractors were digits that occurred between the to-be-remembered words. This could have led the words to be distinctive not only because of their temporal distance from each other, but also because of their status as words, as compared with the neighboring digits. However, it seems unlikely that the contrast between a word and its digit neighbors would extend beyond an all-or-none effect, in contrast to the observed linear effect of temporal interval.

These results thus parallel those found in free recall and with unconstrained reconstruction of serial order information. Our data support the proposition that items are represented in memory in terms of their locations along both a positional and a temporal dimension. The weight given to the positional and temporal dimensions is assumed to depend on task demands (Lewandowsky, Nimmo, \& Brown, 2008). In forward serial recall, positional information is crucial, and late-presented items cannot benefit from temporal/contextual overlap, because such items are necessarily reported last, by which time they will have receded into the temporally crowded past. More weight will therefore be put on the positional, rather than on the temporal, dimension. In contrast, free recall and recognition tasks do not require recall of positional information, and in addition, late-presented items can benefit from early retrieval. Accordingly, it is adaptive to place more weight on the temporal dimension in these tasks. The same rationale applies for unconstrained reconstruction tasks, in which people must retain and retrieve order information but are free to do so in any order. In confirmation, Lewandowsky, Nimmo, and Brown observed that people preferentially reported isolated items earlier than temporally crowded items.

The fact that a TIE was observed only for remember responses appears consistent with the suggestion that recollective processes, akin to those involved in free recall, involve a temporal dimension, whereas familiarity processes do not. As was noted above, it is not universally accepted that dual-process views underpin remember/ know judgments, but the empirically observed TIEs do not depend on acceptance of particular recognition models. We note that alternatives to dual-process models typically predict smaller effects of experimental variables for lowconfidence (know) than for high-confidence (remember) responses (Dunn, 2004); our data are as compatible with this expectation of single-process views as they are with dual-process views.

We next present an experiment that resolved two other issues left open by previous research on TIEs in serial recall. First, as was noted above, all the studies showing no significant TIEs have used articulatory suppression (i.e., repeated articulation of a single word) to prevent rehearsal. Second, in those previous studies, the items presented were taken from a limited pool (a closed set).

\section{EXPERIMENT 2}

TIEs were examined using a serial recall task. As in Experiment 1 , and in departure from most precedents, a con- tinual distractor task was used to prevent rehearsal more rigorously. Similarly, as in Experiment 1 and in departure from precedents, an open pool of words was used.

\section{Method}

Participants. Twenty-one 1st-year students from the University of Warwick participated voluntarily and received course credit in exchange. The experiment lasted approximately $50 \mathrm{~min}$. Five participants were dropped from the analyses due to poor performance (less than $25 \%$ correct recall overall).

Apparatus and Materials. The experiment was controlled by E-Prime. Four hundred thirty-four one-syllable words with an average written frequency of $22(S D=13)$ and an average concreteness rating of $506(S D=98)$ were taken from the MRC Psycholinguistic Database (Wilson, 1988). These words were used to construct 62 lists of seven words for 2 practice trials and 60 experimental trials.

Each trial began with the presentation of a cross in the middle of the screen for 2,000 msec, after which the first word was presented. Each word except the last one was followed by one, two, three, five, seven, or nine digits. These gaps were used in each list, but in a different order on each trial. Across trials, each gap was presented equally often at each serial position. The words and the digits were presented at the rate of $550 \mathrm{msec} / \mathrm{item}$ and were read aloud by the participants. Following the last item and after a $400-\mathrm{msec}$ pause, a series of asterisks was presented to signal recall. The participants then orally recalled the words in their presentation order. If the participants could not remember a word at a particular position, they were asked to say blank for that position. The total list presentation time was $21.1 \mathrm{sec}$

Procedure. The participants were tested individually in a soundattenuating booth. The experimenter was present throughout the experiment to ensure that the participants read the words and digits aloud during presentation of the list and to record the participants' oral recall.

\section{Results}

Accuracy of recall as a function of serial position is shown in Figure 3. The usual serial position curve is evident for the serial recall task when items are read aloud. The slope for performance on the first three items (primacy) and the last three items (recency) was calculated for each participant and subjected to analysis. As can be observed in Figure 3, the primacy part of the curve showed a decrease in performance across serial positions $[M=$ $-.09, S D=.06 ; t(15)=-6.31, p<.001, d=1.73]$, whereas the recency portion of the curve showed an increase in performance across serial positions $[M=.16$, $S D=.09 ; t(15)=6.89, p<.001, d=1.72]$.

In order to analyze the effect of temporal isolation, the combined temporal isolation (time before an item + time after an item) for Positions 2, 4, and 6 was computed. Positions 1 and 7 could not be used because they were not preceded or followed by a gap. Moreover, since the gap after an item is also the gap before another, only these three serial positions were used, to avoid using a given gap twice in the analyses. (This precaution was not necessary in Experiment 1 , since only one item was tested in each list.)

As in Experiment 1, the combined temporal isolations were collapsed into groups containing a similar number of observations (specifically, temporal isolations of 3-5, $6-8,9-11$, and $12-16$; 550-msec digits were collapsed together), and the slope of the resulting function was calculated for each participant. The mean slope (Figure 2) 


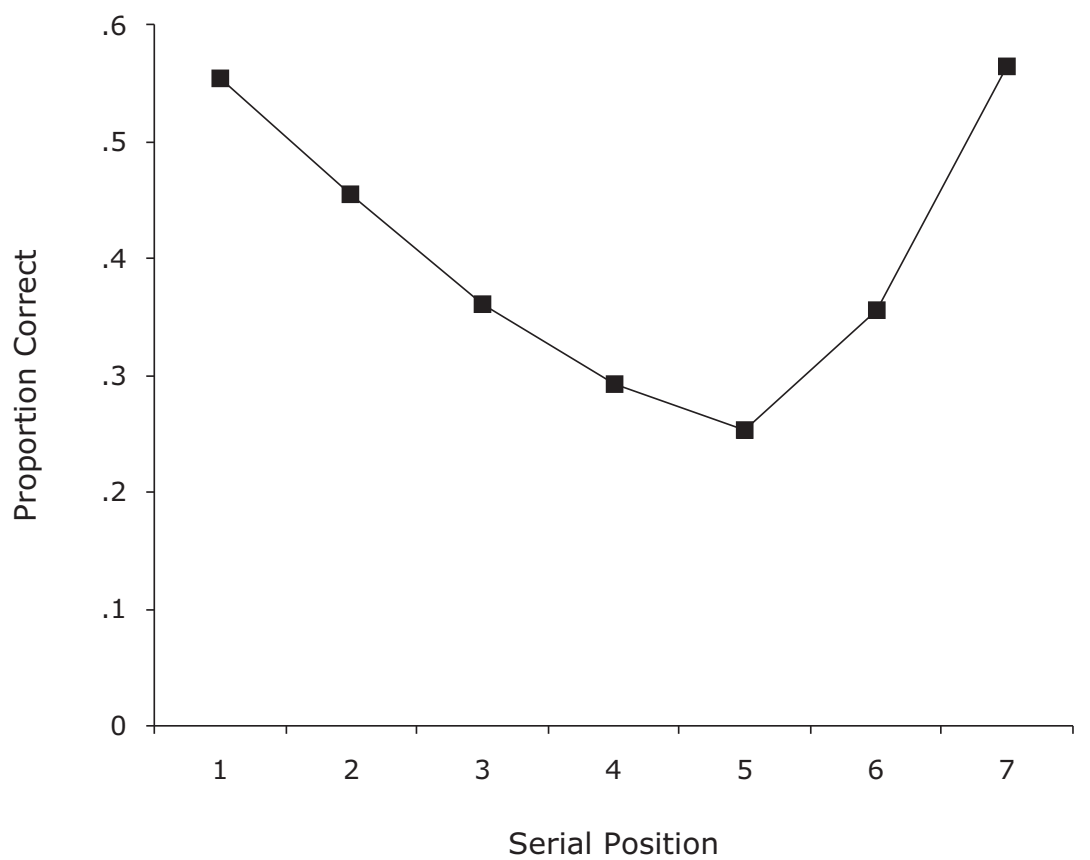

Figure 3. Serial position curve for Experiment 2.

was significantly different from zero $[M=.01, S D=.02$; $t(15)=2.60, p=.02, d=0.65]$ and showed an improvement in correct recall of about $1 \%$ for each additional second of temporal isolation. The mean accuracy of around $35 \%$ after about $2 \mathrm{sec}$ of isolation increased to around $40 \%$ after more than $7 \mathrm{sec}$ of temporal isolation. The effect was thus considerably smaller than that in Experiment 1 . In order to verify that the effect of temporal isolation was still significant when time from item offset was partialed out, a separate logistic regression for each participant was run on the strict serial recall data, with temporal isolation and time from item offset as predictors. The resulting coefficients were submitted to analysis, which revealed that a significant effect of temporal isolation remained $[M=$ $.02, S D=.04 ; t(15)=2.5, p=.03, d=0.63]$.

An additional analysis examined the temporal gaps associated with the first incorrectly recalled item on each trial. ${ }^{1}$ This analysis was motivated by the possibility that "knock-on" effects might occur after the first error, potentially obscuring the effects of temporal isolation. Such analyses must be treated with caution, due to the nonindependence of items' temporal isolations; a late-list item that is relatively temporally isolated will have a slight tendency to be preceded by items that are less temporally isolated, and vice versa. It is nonetheless reassuring that the analysis confirmed a clear effect of temporal isolation on recall of a given item when all the previous items had been recalled correctly. For each of the six possible preitem and postitem intervals, we summed across participants the number of times an item preceded (or followed) by that interval was recalled incorrectly as the first error as a proportion of the number of times it could have been recalled incorrectly as the first error. Separate regressions for preitem and postitem gaps showed that longer intervals led to better performance in both cases (for preitem gaps, $B=.016, S E=$ $.005, t=3.07, p=.037, R^{2}=.70$; for postitem gaps, $B=$ $.019, S E=.004, t=4.69, p=.009, R^{2}=.85$ ). (Note that, unlike earlier analyses, this result applies to data collapsed across participants, as well as across serial positions.)

\section{Discussion}

Results showed that a small but significant TIE can be obtained in a forward serial recall task. The size of the increase in recall for each additional second was smaller than has been observed in free recall (Brown et al., 2006) or recognition (Experiment 1), but the effect was nonetheless significant. The recall advantage increased with temporal isolation, suggesting that the local distinctiveness conferred on to-be-remembered items by being surrounded by the (dissimilar) distractors was not responsible for the effect. Importantly, however, the size of the isolation effect was similar to those observed (albeit nonsignificantly in the statistical analyses) in other forward serial recall experiments. We take up the issue of effect sizes in the General Discussion section.

\section{GENERAL DISCUSSION}

This article addressed three key questions. The first question was whether a TIE would be found in a recognition task, as predicted by temporal distinctiveness models. Experiment 1 showed that isolation effects did indeed influence the probability of making remember, although not know, responses in a recognition task. 
The second question was whether TIEs might be found in forward serial recall when a stringent rehearsal prevention manipulation was used along with an open pool of items. A small but statistically significant isolation effect was indeed obtained. However, despite its statistical significance, the magnitude of the isolation effect was smaller than that seen in tasks such as free recall (Brown et al., 2006), and we therefore now turn to the third question addressed by this article, by examining TIEs in different experimental paradigms.

We start with two empirical generalizations regarding TIEs in memory for verbal material. First, most studies involving tasks other than strictly forward serial recall have shown TIEs. Thus, the effects are seen in unconstrained reconstruction-of-order tasks (Lewandowsky, Nimmo, \& Brown, 2008), free recall tasks (Brown et al., 2006), running memory span (Geiger \& Lewandowsky, 2008), and recognition tasks (this article). In contrast, most studies of forward serial order memory have not shown isolation effects when rehearsal was prevented and random temporal presentation was used (Farrell, 2008; Lewandowsky \& Brown, 2005; Lewandowsky et al., 2006; Lewandowsky, Nimmo, \& Brown, 2008; Nimmo \& Lewandowsky, 2005, 2006; Parmentier et al., 2006). Furthermore, Farrell and McLaughlin (2007) did not find any effect of temporal isolation in a serial recognition task. In deviation from this overall pattern, two studies of serial recall have shown a small but statistically significant TIE (Experiment 3 in Farrell, 2008, and Experiment 2 in the present article 2 ).

One possible account of this overall pattern is that forward serial recall generally is characterized by a very small isolation effect that, on occasion, is statistically detectable. To assess this possibility, we used nonparametric tests to examine directions of TIEs - expressed as facilitation of performance per second of additional isolation-across all available studies of verbal memory in which TIEs were reported. ${ }^{3}$ We considered 19 different conditions or studies for forward serial recall and 7 conditions/studies for all the other tasks (free recall, recognition, etc.). The results are shown as a pair of histograms in Figure 4. Each panel shows the effect size distributions for forward serial recall tasks (unfilled bars; top panel) and all other tasks (shaded bars; bottom panel). It is immediately evident that (1) most of the 19 effects for forward serial recall were positively signed, a result unlikely to be due to chance alone, and (2) there was a strong tendency for effects to be greater in tasks other than forward serial recall.

Effect magnitudes were taken from mean slopes (relating recall probability to absolute temporal isolation measured in seconds) reported in the literature. We ignored the potentially complicating factor that relative, rather than absolute, temporal isolation might be the relevant factor, although preliminary investigations suggested that this assumption had little effect on the conclusions. ${ }^{4}$ When slope values were reported separately for preitem and postitem gaps, they were averaged for our analysis. We excluded all the conditions in which an explicit instruction to group was involved but included cases in which spontaneous grouping of the list may have occurred. Finally, in cases in which logistic regression was used or the parameter values could not be straightforwardly compared for other reasons, we estimated slopes from reported graphs or data provided to us by the authors. Slopes were calculated directly in this way to obtain the effects sizes from Parmentier et al. (2006), Brown et al. (2006; free recall), and Experiments 2 and 3 of Farrell (2008). ${ }^{5}$ Slopes thus obtained were sometimes estimated from as few as three data points and cannot be reliably interpreted individually. In addition, precise slope magnitudes may depend on the binning of temporal isolations employed in different studies. However, although any individual data point should be treated with considerable caution, the overall pattern seems remarkably clear. Seventeen of the 19 effects for forward serial recall are positive (sign test: $p<.001$ ). Furthermore, effects are smaller for forward serial recall tasks than for other tasks: All 7 of the effects for tasks other than forward serial recall are greater than the mean of the forward serial recall effects ( $\operatorname{sign}$ test: $p<.05$ ).

The analysis just described examined total temporal isolation and left open the possibility that the effects might reflect an effect solely of postitem temporal intervals (in which covert rehearsal or consolidation might occur). We therefore examined all published cases in which effects of preitem and postitem gaps were reported separately. Effects for different serial positions, where available, were treated as separate observations. For postitem temporal intervals, we found 18 positively signed and 5 negatively signed effects (sign test: $p<.01$ ). For preitem temporal intervals, we found 14 positively signed and 7 negatively signed effects (sign test: $.05<p<.10$ ). If individual participant effects are treated as separate observations, both preitem and postitem effects are independently significant. These analyses provide some reassurance that the apparent overall effect of temporal isolation is not solely due to processes occurring after item presentations but leave open the possibility that longer temporal gaps may lead to better performance due to greater grouping (Farrell, Lelièvre, \& Wise, 2009).

At an empirical level, the conclusion seems clear. TIEs are substantially smaller in forward serial recall than in other tasks but, overall, are greater than zero. Indeed, in forward serial recall, the effect size is sufficiently small that it has failed to reach statistical significance in most experiments.

Overall, the pattern of results appears to cause difficulty for memory models that accord no role to time. Before interpreting this pattern within the framework of temporal distinctiveness models, we note that the requirement for forward serial recall is not the only difference between forward serial recall and other memory tasks. In particular, list lengths are generally shorter in studies of serial recall than in recognition and free recall experiments, raising the possibility that TIEs might occur only at longer list lengths. List length cannot be the only factor determining the size of TIEs, however, because TIEs can either be found or not found as a function of a postcuing manipulation in which list length is necessarily constant (Lewandowsky, Nimmo, 

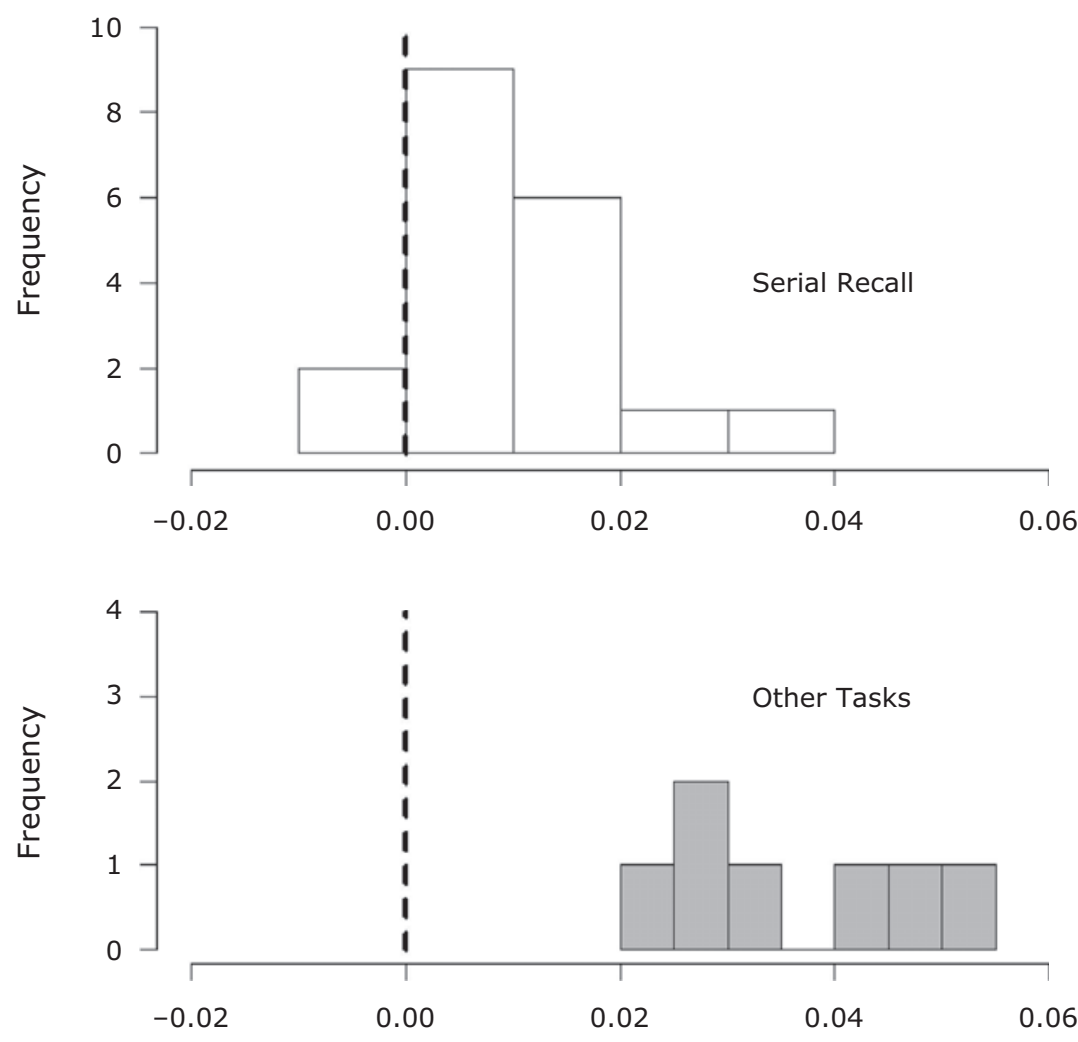

Isolation Effect

Figure 4. Magnitudes of temporal isolation effects. The parameter estimates for the forward serial recall experimental conditions were taken from Nimmo and Lewandowsky (2005; both quiet and suppression conditions), Lewandowsky, Brown, Wright, and Nimmo (2006; both quiet and suppression conditions and probed recall Experiment 2), Nimmo and Lewandowsky (2006; Experiment 1, short and long; Experiment 2, auditory and visual), Lewandowsky, Nimmo, and Brown (2008; all three forward serial recall conditions), Lewandowsky (unpublished; probed serial recall, as cited in Geiger \& Lewandowsky, 2008), Parmentier, King, and Dennis (2006), Lewandowsky and Brown (2005; both experiments), Farrell (2008; Experiments 2 and 3, ungrouped conditions), and the present Experiment 2. The parameter estimates for other tasks were taken from Brown, Morin, and Lewandowsky (2006; free recall), Lewandowsky, Nimmo, and Brown (2008; all three free serial order reconstruction conditions), Geiger and Lewandowsky (2008; both running memory span experiments), and the present Experiment 1.

\& Brown, 2008). It remains unknown, however, whether TIEs would disappear in a free recall task when short list lengths are used, since it is known that free recall tends to occur in forward order under such conditions (Ward, Tan, \& Grenfell-Essam, 2010).

Lewandowsky et al. (2009) suggested that forward serial recall can be seen as a ballistic process, in that once the first item has been produced, recall proceeds in an automatic fashion without the possibility of strategic intervention (e.g., to decide the order of recall). All the other tasks (except recognition, where just one item is involved) allow participants to decide which item to recall first and which items to recall subsequently. This consideration also applies to the running memory span task (Geiger \& Lewandowsky, 2008), notwithstanding the use of forward recall, because people must first locate the initial to-berecalled item when list presentation unexpectedly stops. The ballistic nature of serial recall may therefore set it aside from other retrieval tasks.

We interpret this pattern of results within the SIMPLE framework (Brown et al., 2007). According to this model, items are represented in a multidimensional memory space, in which two important dimensions are the positional and temporal dimensions. To the extent that items are represented along a temporal (rather than a positional or another) dimension, TIEs will be observed. Items are more likely to be retrieved on the basis of their position along a temporal dimension when the nature of the task renders such a representation useful. Broadly speaking, this will occur whenever recently presented items can be 
recalled early, thus taking advantage of their temporal recency (see Lewandowsky et al., 2009, for an extensive discussion). Thus it is adaptive- just in the sense of leading to better performance-for participants to give less weight to the temporal dimension during retrieval when recent items cannot be recalled early to take advantage of temporal/contextual overlap. ${ }^{6}$ Forward serial recall is one of the few tasks in which late-presented items will not benefit from their recency, because such items must be recalled after the time-consuming recall of earlier-presented items. It therefore seems likely that less attentional weight is given to a temporal dimension during retrieval in purely forward serial recall and that the smaller TIEs seen in forward serial recall reflect this adaptive processing.

\section{AUTHOR NOTE}

Preparation of this article was facilitated by ESRC Grant RES000231038 to the first and second authors, a Discovery Grant from the Australian Research Council and an Australian Professorial Fellowship to the third author, and a Linkage International Grant from the Australian Research Council to the second and third authors. Address correspondence to C. Morin, Department of Informatics and Sensors, Cranfield University, Shrivenham, Swindon SN6 8LA, England (e-mail: c.morin@cranfield.ac.uk).

\section{REFERENCES}

Barrouillet, P., Bernardin, S., Portrat, S., Vergaume, E., \& Camos, V. (2007). Time and cognitive load in working memory. Journal of Experimental Psychology: Learning, Memory, \& Cognition, 33, 570-585.

BJork, R. A., \& Whitten, W. B. (1974). Recency-sensitive retrieval processes in long-term free recall. Cognitive Psychology, 6, 173189.

Brown, G. D. A., \& Lewandowsky, S. (2010). Forgetting in memory models: Arguments against trace decay and consolidation failure. In S. Della Sala (Ed.), Forgetting (pp. 49-75). Hove, U.K.: Psychology Press.

Brown, G. D. A., Morin, C., \& Lewandowsky, S. (2006). Evidence for time-based models of free recall. Psychonomic Bulletin \& Review, 13, 717-723.

Brown, G. D. A., Neath, I., \& Chater, N. (2007). A temporal ratio model of memory. Psychological Review, 114, 539-576.

Brown, G. D. A., Preece, T., \& Hulme, C. (2000). Oscillator-based memory for serial order. Psychological Review, 107, 127-181.

Brown, G. D. A., Vousden, J. I., \& McCormack, T. (2009). Memory retrieval as temporal discrimination. Journal of Memory \& Language, 60, 194-208.

Corballis, M. C. (1966). Memory span as a function of variable presentation speeds and stimulus durations. Journal of Experimental Psychology, 71, 461-465

Crowder, R. G. (1976). Principles of learning and memory. Hillsdale, NJ: Erlbaum.

Crowder, R. G., \& Neath, I. (1991). The microscope metaphor in human memory. In S. Lewandowsky \& W. E. Hockley (Eds.), Relating theory and data: Essays on human memory in honor of Bennet B. Murdock (pp. 111-125). Hillsdale, NJ: Erlbaum.

DunN, J. C. (2004). Remember-know: A matter of confidence. Psychological Review, 111, 524-542.

FARRELL, S. (2008). Multiple roles for time in short-term memory: Evidence from serial recall of order and timing. Journal of Experimental Psychology: Learning, Memory, \& Cognition, 34, 128-145.

Farrell, S., Lelièvre, V., \& Wise, A. (2009). Are timing effects on serial recall modulated by grouping? Unpublished manuscript.

FarReLl, S., \& LEWANDOWSKY, S. (2002). An endogenous distributed model of ordering in serial recall. Psychonomic Bulletin \& Review, 9, 59-79.

FarReLL, S., \& MCLAughlin, K. (2007). Short-term recognition memory for serial order and timing. Memory \& Cognition, 35, 1724-1734.
Geiger, S. M., \& Lewandowsky, S. (2008). Temporal isolation does not facilitate forward serial recall—or does it? Memory \& Cognition, 36, 957-967.

Guérard, K., Neath, I., Surprenant, A. M., \& Tremblay, S. (2010). Distinctiveness in serial memory for spatial information. Memory \& Cognition, 38, 83-91.

Jones, D. M., \& MACKEN, W. J. (1993). Irrelevant tones produce an irrelevant speech effect: Implications for phonological coding in working memory. Journal of Experimental Psychology: Learning, Memory, \& Cognition, 19, 369-381.

Knoedler, A. J., Hellwig, K. A., \& Neath, I. (1999). The shift from recency to primacy with increasing delay. Journal of Experimental Psychology: Learning, Memory, \& Cognition, 25, 474-487.

LEWANDOWSKy, S., \& BRown, G. D. A. (2005). Serial recall and presentation schedule: A micro-analysis of local distinctiveness. Memory, 13, 283-292.

Lewandowsky, S., Brown, G. D. A., \& Thomas, J. L. (2009). Traveling economically through memory space: Characterizing output order in memory for serial order. Memory \& Cognition, 37, 181193.

Lewandowsky, S., Brown, G. D. A., Wright, T., \& Nimmo, L. M. (2006). Timeless memory: Evidence against temporal distinctiveness models of short-term memory for serial order. Journal of Memory \& Language, 54, 20-38.

Lewandowsky, S., Duncan, M., \& Brown, G. D. A. (2004). Time does not cause forgetting in short-term serial recall. Psychonomic Bulletin \& Review, 11, 771-790.

Lewandowsky, S., Geiger, S. M., \& Oberauer, K. (2008). Interference-based forgetting in verbal short-term memory. Journal of Memory \& Language, 59, 200-222.

Lewandowsky, S., \& Murdock, B. B. (1989). Memory for serial order. Psychological Review, 96, 25-57.

Lewandowsky, S., Nimmo, L. M., \& Brown, G. D. A. (2008). When temporal isolation benefits memory for serial order. Journal of Memory \& Language, 58, 415-428.

Lewandowsky, S., Wright, T., \& Brown, G. D. A. (2007). The interpretation of temporal isolation effects. In N. Osaka, R. H. Logie, \& M. D'Esposito (Eds.), The cognitive neuroscience of working memory (pp. 137-152). Oxford: Oxford University Press.

Macken, W. J., \& Jones, D. M. (1995). Functional characteristics of the inner voice and the inner ear: Single or double agency? Journal of Experimental Psychology: Learning, Memory, \& Cognition, 21, 436-448.

Nairne, J. S., Neath, I., Serra, M., \& Byun, E. (1997). Positional distinctiveness and ratio rule in free recall. Journal of Memory \& Language, 37, 155-166.

Neath, I. (1993a). Contextual and distinctive processes and the serial position function. Journal of Memory \& Language, 32, 820-840.

Neath, I. (1993b). Distinctiveness and serial position effects in recognition. Memory \& Cognition, 21, 689-698.

Neath, I., \& Crowder, R. G. (1990). Schedules of presentation and distinctiveness in human memory. Journal of Experimental Psychology: Learning, Memory, \& Cognition, 16, 316-327.

Neath, I., \& Crowder, R. G. (1996). Distinctiveness and very shortterm serial position effects. Memory, 4, 225-242.

Neath, I., \& Knoedler, A. J. (1994). Distinctiveness and serial position effects in recognition and sentence processing. Journal of Memory \& Language, 33, 776-795.

Nimmo, L. M., \& Lewandowsky, S. (2005). From brief gaps to very long pauses: Temporal isolation does not benefit serial recall. Psychonomic Bulletin \& Review, 12, 999-1004.

Nimmo, L. M., \& Lewandowsky, S. (2006). Distinctiveness revisited: Unpredictable temporal isolation does not benefit short-term serial recall of heard or seen events. Memory \& Cognition, 34, 13681375 .

Oberauer, K., \& Lewandowsky, S. (2008). Forgetting in immediate serial recall: Decay, temporal distinctiveness, or interference? Psychological Review, 115, 544-576.

Page, M. P. A., \& NorRIS, D. (1998). The primacy model: A new model of immediate serial recall. Psychological Review, 105, 761-781.

Parmentier, F. B. R., King, S., \& DenNis, I. (2006). Local temporal distinctiveness does not benefit auditory verbal and spatial serial recall. Psychonomic Bulletin \& Review, 13, 458-465. 
Schonfield, D., \& Donaldson, W. (1966). Immediate memory as a function of intraseries variation. Canadian Journal of Psychology, 20, 218-227.

Talmi, D., \& Goshen-Gottstein, Y. (2006). The long-term recency effect in recognition memory. Memory, 14, 424-436.

WARD, G., TAN, L., \& GRENFELl-Essam, R. (2010). Examining the relationship between free recall and immediate serial recall: The effects of list length and output order. Journal of Experimental Psychology: Learning, Memory, \& Cognition, 36, 1207-1241.

Welte, J. W., \& LaUgherY, K. R. (1971). Short-term memory: The effects of interitem time distribution and recall procedure. Canadian Journal of Psychology, 25, 436-442.

WILson, M. D. (1988). The MRC Psycholinguistic Database: Machineusable dictionary, version 2.00. Behavior Research Methods, Instruments, \& Computers, 20, 6-10.

WiXTED, J. T. (2007). Dual-process theory and signal detection theory of recognition memory. Psychological Review, 114, 152-176.

YonelinAs, A. P. (2002). The nature of recollection and familiarity: A review of 30 years of research. Journal of Memory \& Language, 46, 441-517.

\section{NOTES}

1. We are grateful to Geoff Ward for suggesting this analysis.

2. Experiment 3 of Farrell (2008) differed from the present Experiment 2 primarily in that (1) the former involved memory for digits, not words, (2) auditory presentation was used, and (3) no attempt was made to prevent rehearsal.

3 . We thus excluded one study of memory for nonverbal material (Parmentier et al., 2006), while noting here that a negative effect of temporal isolation was found in that study, and excluded another (Guérard et al., 2010) that showed a positive TIE.

4. For example, consider two conditions of a hypothetical memory experiment. In Condition A, half the items have a total temporal isolation of $10 \mathrm{sec}$, and the other half of the items have a total temporal isolation of $11 \mathrm{sec}$. In Condition B, half the items have a total temporal isolation of $0.1 \mathrm{sec}$, and the other half of the items have a total temporal isolation of $1.1 \mathrm{sec}$. It seems clear that the recall advantage for the more isolated items will be much greater in Condition B (in which the isolated items are 11 times more isolated than their neighbors) than in Condition A (in which the isolated items are just $10 \%$ more isolated). But the increase in isolation between the less isolated and more isolated items is the same (if measured in absolute time) in both cases.

5. We thank Simon Farrell for providing these data.

6. Applications of the SIMPLE model have hitherto assumed that the attentional weight given to a temporal dimension cannot vary during the course of retrieval, but this assumption remains untested.

(Manuscript received September 30, 2009; revision accepted for publication March 19, 2010.) 